\title{
Adaptive immune cells temper initial innate responses
}

\author{
Kwang Dong Kim ${ }^{1-3}$, Jie Zhao ${ }^{1,3}$, Sogyong $\mathrm{Auh}^{2}$, Xuanming Yang ${ }^{1}$, Peishuang $\mathrm{Du}^{1}$, Hong Tang ${ }^{1}$ \& \\ Yang-Xin $\mathrm{Fu}^{1,2}$
}

Toll-like receptors (TLRs) recognize conserved microbial structures called pathogen-associated molecular patterns. Signaling from TLRs leads to upregulation of co-stimulatory molecules for better priming of $\mathrm{T}$ cells and secretion of inflammatory cytokines by innate immune cells ${ }^{1-4}$. Lymphocytedeficient hosts often die of acute infection, presumably owing to their lack of an adaptive immune response to effectively clear pathogens. However, we show here that an unleashed innate immune response due to the absence of residential T cells can also be a direct cause of death. Viral infection or administration of poly(I:C), a ligand for TLR3, led to cytokine storm in T-cell- or lymphocyte-deficient mice in a fashion dependent on NK cells and tumor necrosis factor. We have further shown, through the depletion of $\mathrm{CD}^{+}$and $\mathrm{CD}^{+}$cells in wild-type mice and the transfer of $T$ lymphocytes into Rag-1deficient mice, respectively, that $T$ cells are both necessary and sufficient to temper the early innate response. In addition to the effects of natural regulatory $T$ cells, close contact of resting CD4 ${ }^{+}$CD25-Foxp3- $3^{-}$or CD8 $^{+}$T cells with innate cells could also suppress the cytokine surge by various innate cells in an antigen-independent fashion. Therefore, adaptive immune cells have an unexpected role in tempering initial innate responses.

To study the early innate response, we infected nude mice and wildtype mice with MHV-A59, a coronavirus that primarily infects mouse liver and brain ${ }^{5,6}$. Nude mice died at a sublethal dose of virus as compared with wild-type mice (Fig. 1a). We presumed that lack of $\mathrm{T}$ cells might permit vigorous progression of the viral infection, enough to kill the host. To our surprise, the virus titer in the liver was not significantly higher in nude mice than in wild-type mice (Fig. 1b). To determine the cause of death, we collected liver and brain tissues and assessed them by H\&E staining, but we did not observe any major pathology (data not shown). As a functional readout for organ damage after infection, we measured the concentrations of alanine aminotransferase (ALT) and aspartate aminotransferase (AST), but we detected only mild increases in nude mice as compared to wild-type mice, insufficient to explain the elevated death rate of the former (Supplementary Fig. 1). This raised the intriguing possibility that the immunocompromised mice might actually die due to cytokine storm. To test that, the sera of mice were collected and cytokine levels were determined on days 2 and 4 . Higher abundances of proinflammatory cytokines were detected in T cell-deficient mice than in wild-type mice $(n=16)$ on both day 2 (Fig. 1c and Supplementary Fig. 2) and day 4 (data not shown). The data suggest that acute infection in immunocompromised mice might result in stronger innate immune responses.

MHV-A59 is an RNA virus that can target TLR3, and RNA virusrelated poly(I:C) is a noninfectious ligand for TLR3 (ref. 7). To analyze susceptibility to TLR3 stimulation independent of infectious particles, we used the poly(I:C) compound to ensure that both wild-type and immunocompromised mice were exposed to similar levels of TLR3 stimulation. Notably, all nude mice rapidly died in 12-24 h after receiving a sublethal dose of poly(I:C) (Fig. 2a). As seen in MHV-A59 infection, various proinflammatory cytokine levels were much higher in nude mice than wild-type mice at 2 and $6 \mathrm{~h}$ after treatment (Fig. 2b and Supplementary Fig. 3a). It is likely that this rapid death of the mice could be attributable to cytokine storm, similar to that caused by lipopolysaccharide (LPS) or superantigens ${ }^{8-10}$. To study whether other immunocompromised mice have similar sensitivity to poly(I:C), we used Rag-1 knockout mice, which lack functional lymphocytes. Like nude mice, Rag-1 knockout mice also showed hypersensitivity to poly(I:C) and died within 12-24 h after treatment with a sublethal dose of the compound (Fig. 2c). Again, cytokine levels were much higher in the Rag-1 knockout mice than in wild-type mice (Fig. 2d and Supplementary Fig. 3b). To further determine whether T cells are essential to control the early innate response, we depleted wild-type mice with antibodies to CD4 and CD8 before poly(I:C) treatment, and found that the depleted mice produced more inflammatory cytokines than the control mice (Fig. 2e). To assess whether T cells are sufficient to control the overzealous cytokine response, we adoptively transferred lymphocytes to Rag-1 knockout mice, and after $2 \mathrm{~d}$ treated them with poly(I:C). The reconstituted mice produced lower levels of tumor necrosis factor (TNF) in $2 \mathrm{~h}$, and interferon (IFN) $\gamma$ in $6 \mathrm{~h}$, than control mice (Fig. 2f). It is possible that low-grade infection in these immunodeficient mice might cause more activation of innate cells. To examine whether $\mathrm{T}$ cells are sufficient to temper the initial cytokine surge of innate cells from the same source, we divided splenocytes from wild-type mice into non- $\mathrm{T}$ and $\mathrm{T}$ cell populations. Non-T cells in culture responded vigorously to MHV-A59 or poly(I:C) to produce high levels of cytokines, whereas T cells did not. Notably, addition of $\mathrm{T}$ cells to non-T cells efficiently prevented this cytokine surge (Fig. 3a and Supplementary Fig. 4). This suggests that constant

${ }^{1}$ Center for Infection and Immunity and National Laboratory of Biomacromolecules, Institute of Biophysics, Chinese Academy of Sciences, 15 Da Tun Road, Chaoyang District, Beijing 100101, China. ${ }^{2}$ Department of Pathology, University of Chicago, Chicago, Illinois 60637, USA, ${ }^{3}$ These authors contributed equally to this work. Correspondence should be addressed to H.T. (tanghong@moon.ibp.ac.cn) or Y.-X.F. (yfu@uchicago.edu).

Received 27 March; accepted 31 July; published online 23 September 2007; doi:10.1038/nm1633 

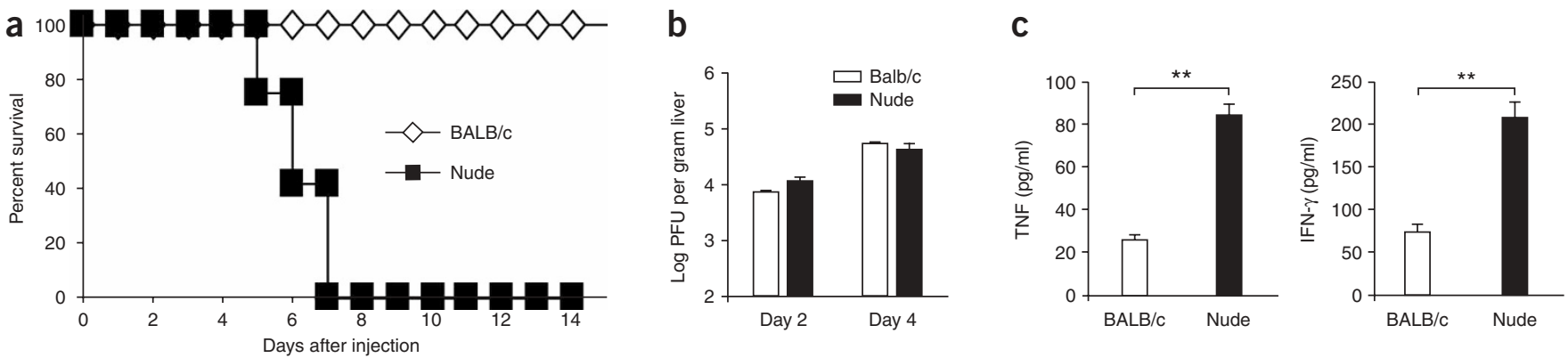

Figure 1 Comparison of mortality rates, liver virus titers and serum inflammatory cytokines of wild-type BALB/c mice and nude mice after MHV-A59 infection. (a) Mortality curves of wild-type BALB/c $(\diamond, n=12)$ and nude mice $(\mathbf{\square}, n=12)$ after infection with $4 \times 10^{5} \mathrm{PFU}$ MHV-A59. (b) Liver virus titers were determined at day $2(P=0.051)$ and day $4(P=0.925)$ after infection $(n=11)$. (c) TNF and IFN- $\gamma$ concentrations in serum were determined at day 2 after infection (pool of 14-16 mice in each group from various experiments). Horizontal bars indicate the median values. Statistical comparisons were significant. ${ }^{*} P<0.01$, by $t$-test.

monitoring by $\mathrm{T}$ cells is required to temper the innate response. To further test which subset of $\mathrm{T}$ cells is more important, we separated $\mathrm{T}$ cells into $\mathrm{CD}^{+}$and $\mathrm{CD}^{+}$populations. When either population of $\mathrm{T}$ cells was mixed with non-T cells, cytokine production was reduced (Fig. $\mathbf{3 b}$ ).

To study whether T-cell receptor (TCR) engagement by specific antigen is required, we used unprimed T cells from OTI $\left(\mathrm{CD}^{+} \mathrm{T}\right.$ cells for ovalbumin (OVA)) or OTII (CD4 ${ }^{+} \mathrm{T}$ cells for OVA) mice (Fig. 3c). Either cellular population could control the cytokine surge, suggesting that the tempering of the innate response is likely to be antigen independent. Because cell-cell contact could still be important in this process, we used a Transwell system, with non-T cells stimulated with poly(I:C) in the lower well and T cells in the upper well, and found that the inhibitory effect was greatly reduced (Fig. 3d). Therefore, T cell-mediated inhibition of the innate response is dependent on cellcell contact. To determine whether contact-dependent functions of $\mathrm{T}$ cells are mediated by TCR-major histocompatibility complex
(MHC) recognition, $\mathrm{CD}^{+}$cells were added into non-T cells from MHC class II knockout mice and the cells were stimulated with poly(I:C) overnight. These class II-deficient non-T cells were only weakly inhibited by $\mathrm{CD} 4^{+} \mathrm{T}$ cells, whereas $\mathrm{CD} 4^{+} \mathrm{T}$ cells from the same source robustly inhibited wild-type non-T cells (Supplementary Fig. 5). The impaired inhibition of class II-deficient non-T cells by $\mathrm{CD}^{+}$cells is consistent with the similarly weak inhibition seen in the Transwell study. Therefore, the inhibition by $\mathrm{T}$ cells is mainly mediated by cell-cell contact and is MHC dependent.

There is increasing evidence implicating regulatory $\mathrm{T}$ cells (Treg) in the regulation not only of other $\mathrm{T}$ cells, but also of innate cells ${ }^{11,12}$. To study whether natural Treg $\left(\mathrm{CD} 4^{+} \mathrm{CD} 25^{+} \mathrm{FoxP} 3^{+}\right)$are essential for the inhibition, we added $\mathrm{CD} 25^{+}$or $\mathrm{CD} 25^{-} \mathrm{T}$ cells into non- $\mathrm{T}$ cells (Fig. 3e). The Treg-depleted T cell population still efficiently inhibited the cytokine surge, much as the whole population of T cells did. Next, we tested the effect of depleting Foxp $3^{+}$cells by using FoxP3(GFP) knock-in mice ${ }^{13}$, whose Foxp $3^{+}$cells have bright green fluorescent $a$
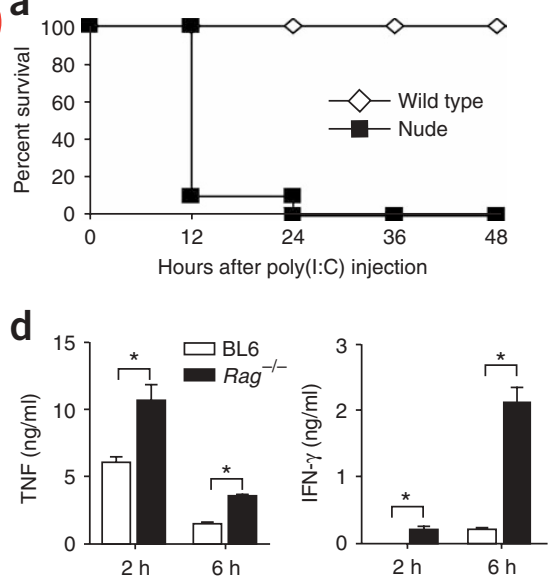
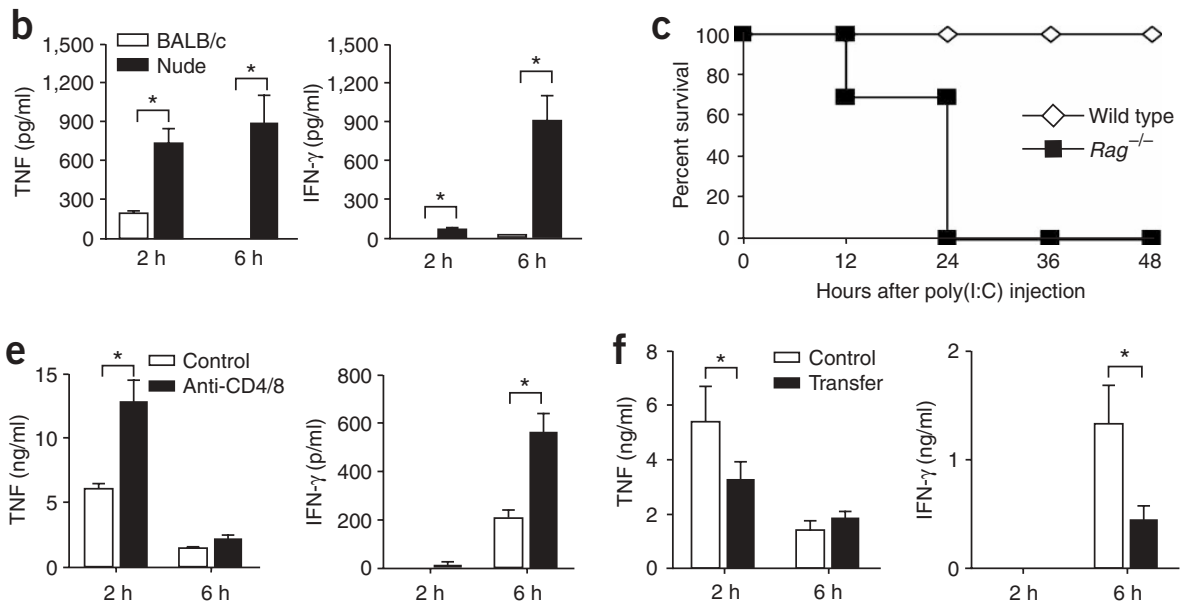

Figure 2 Wild-type mice are more resistant to poly(I:C) than nude mice and Rag-1 knockout mice, and T cells are essential for controlling proinflammatory cytokine production in vivo. (a) Mortality curves of wild-type BALB/c ( $\diamond, n=7)$ and nude mice $(\boldsymbol{\square}, n=10)$ after injection with $700 \mu \mathrm{g}$ poly(I:C). (b) TNF and IFN- $\gamma$ concentrations in serum were determined at $2 \mathrm{~h}$ and $6 \mathrm{~h}$ after poly(I:C) injection ( $n=4-9)$. (c) Mortality curves of wild-type C57BL6 ( $\diamond, n=8)$ and Rag-1 knockout $(\mathbf{\square}, n=16)$ mice after injection with $400 \mu \mathrm{g}$ poly(l:C). (d) TNF and IFN- $\gamma$ in serum of wild-type C57BL6 and Rag-1 knockout mice $2 \mathrm{~h}$ and $6 \mathrm{~h}$ after poly(I:C) injection. (e) TNF and IFN- $\gamma$ in serum of T cell-depleted wild-type C57BL6 mice $2 \mathrm{~h}$ and $6 \mathrm{~h}$ after poly(I:C) injection. The results are representative of two experiments ( $n=6$ mice total, 3 per group). (f) Reduced cytokines in Rag- 1 knockout mice reconstituted with lymphocytes. Rag- 1 knockout mice ( $n=4$ per group) were adoptively transferred with lymphocytes for $2 \mathrm{~d}$ and then treated with poly(I:C); reconstituted mice produced significantly lower levels of cytokines than control mice. ${ }^{*} P<0.05$, by $t$-test. 
a

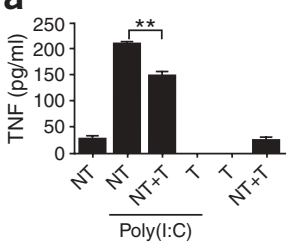

C
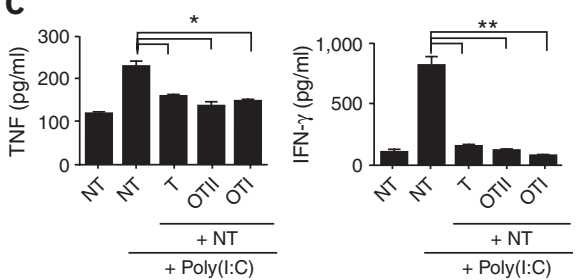

e

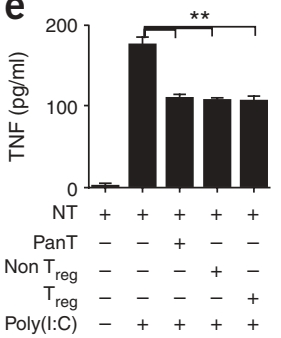

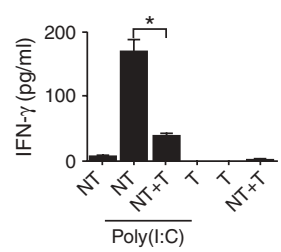

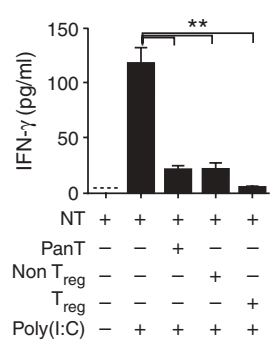

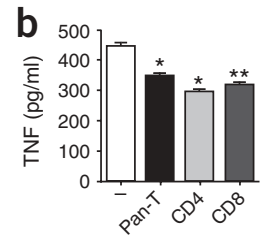
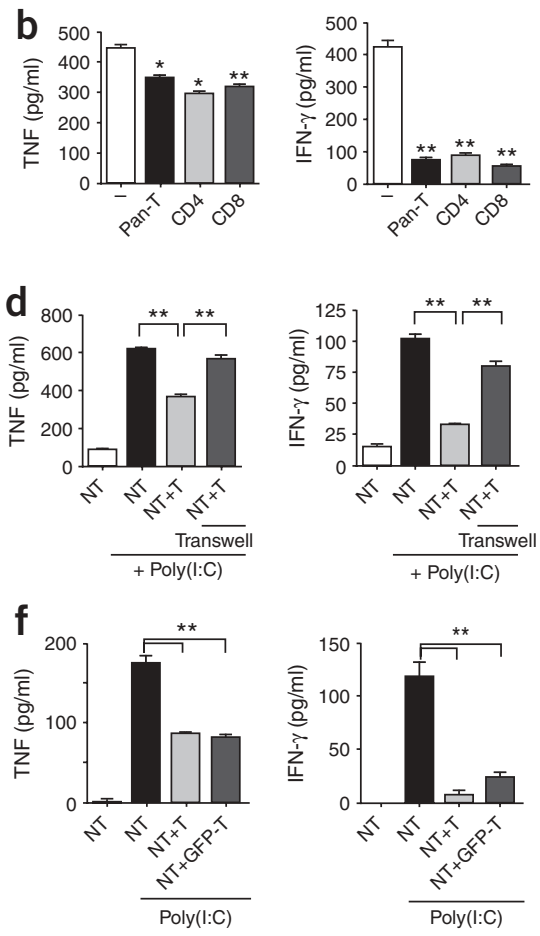

Figure 3 T cells inhibit proinflammatory cytokine production of splenocytes stimulated with poly(I:C) in vitro. (a-c) $1 \times 10^{6}$ non-T cells were stimulated with $100 \mu \mathrm{g} / \mathrm{ml}$ poly(l:C) in the presence or absence of $1 \times 10^{6}$ pan-T (a), CD4 ${ }^{+}$ $\mathrm{T}$ cells or $\mathrm{CD}^{+} \mathrm{T}$ cells $(\mathbf{b})$, or OT-II transgenic $\mathrm{CD}^{+} \mathrm{T}$ cells or OT-I transgenic $\mathrm{CD} 8^{+} \mathrm{T}$ cells (c). (d) To prevent close contacts between non-T cells and $T$ cells, non-T cells were cultured in the lower chamber of a Transwell system containing T cells in the upper chamber. (e,f) $\mathrm{CD} 4^{+} \mathrm{CD} 25^{+}$(Treg) or $\mathrm{CD} 4{ }^{+} \mathrm{CD} 25^{-}$(non-Treg) T cells from wild-type mouse with a ratio of non- $T$ cell to $T$ cell of 1:0.5 (e), or GFP- $\mathrm{T}$ cell (non-Treg) or GFP ${ }^{+}$T cells (Treg) from FoxP3 knock-in mouse (f), were added to non-T cells. The ratio of non-T cells to GFP ${ }^{+}$or GFP- $\mathrm{T}$ cell was 1:0.5. ${ }^{*} P<0.05,{ }^{* *} P<0.01$, by $t$-test. The results are representative of at least two independent experiments for each panel ( $n=6$ per group)

death, as TNFR-hIg treatment rescued these mice, whereas anti-IFN- $\gamma$ did not protect them (Fig. 4c-e and Supplementary Fig. 6). Because NK cells can enhance TNF production by innate cells, we sought to determine the necessity of NK cells in the poly(I:C) response. Rag-1 knockout mice were treated with anti-NK1.1 or anti-asialo-GM-1 antibodies before poly(I:C) injection. After NK-cell depletion, the survival rate of Rag-1 knockout mice was increased to $72 \%$, and their levels of proinflammatory cytokines were corresponding lower (Fig. $4 \mathbf{f}, \mathbf{g})$. TNF and NK cells seem to have essential roles in poly(I:C)-induced sudden death of Rag-1 knockout mice. To test whether $\mathrm{T}$ cells also inhibit other TLR-mediated stimulation on innate cells, we stimulated non-T cells with LPS, a potent stimulator of TLR4, and determined their cytokine levels. Cytokine production by non-T cells was also controlled by addition of T cells (Supplementary Fig. 7). Therefore, $\mathrm{T}$ cells control innate cells under various conditions.

To test whether human $\mathrm{T}$ cells could also inhibit TNF production of human non-T cells stimulated by poly(I:C) and LPS in vitro, we stimulated non-T cells with poly(I:C) or LPS for $30 \mathrm{~min}$. After washing them with PBS, we cocultured these innate cells with or without pan-T cells, CD45RO ${ }^{+} \mathrm{T}$ cells or $\mathrm{CD}^{2} 5 \mathrm{RO}^{-} \mathrm{T}$ cells for $20 \mathrm{~h}$. TNF production was reproducibly suppressed by naive $\mathrm{T}$ cells from various healthy donors (Supplementary Fig. 8). Therefore, T cellmediated inhibition of the innate response may be important for the pathogenesis of acute infection - a finding that suggests a potentially new treatment for human diseases.

Contrary to the principle that lymphocytes might provide positive reinforcement to the ongoing innate response ${ }^{19}$, our study implicates lymphocytes as negative regulators in the very early response to acute infection. The unrestrained innate immune response itself, owing to the lack of supervision by $\mathrm{T}$ cells, can be the direct cause of death from acute infection. In addition to natural regulatory $\mathrm{T}$ cells, large numbers of naive $\mathrm{T}$ cells might be needed to efficiently temper the deadly TLR response at the initial phase of infection. These studies confirm our hypothesis that the adaptive immune system suppresses the innate system in the early phase of viral and nonviral challenges and that lack of $\mathrm{T}$ cells might result in excessive innate responses to TLR stimulation. The concept of an unleashed innate response in the absence of adaptive modulation may also lead to new diagnosis and treatment for individuals with congenital or acquired immune deficiency. 
LETTERS

a
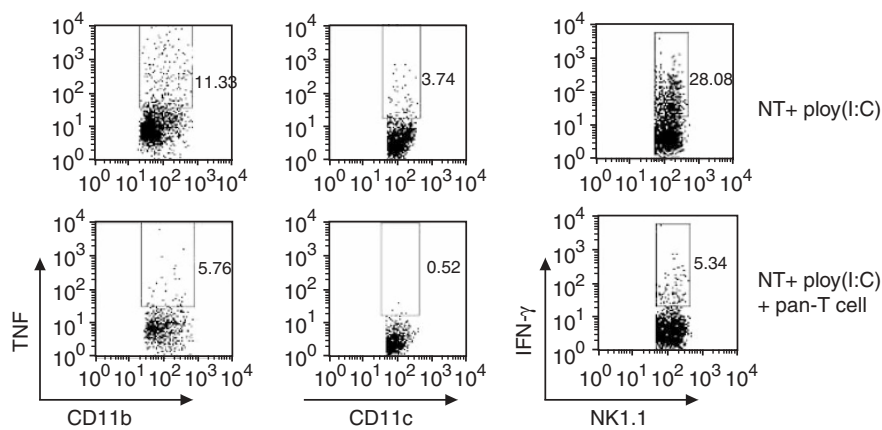

$\mathrm{NT}+$ ploy $(\mathrm{I}: \mathrm{C})$ + pan-T cell b

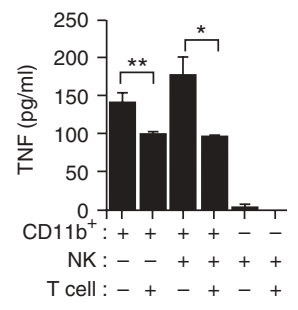

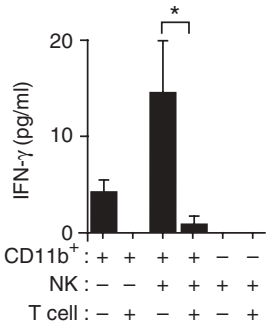

e

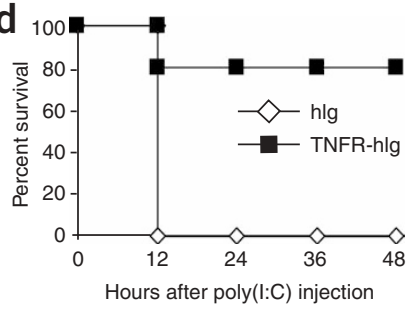

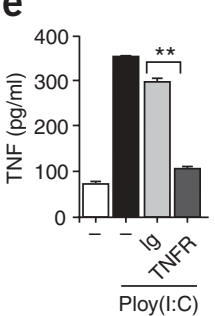

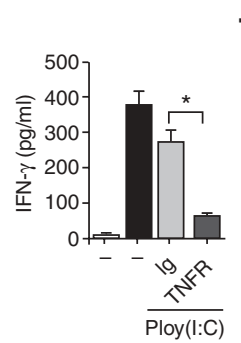

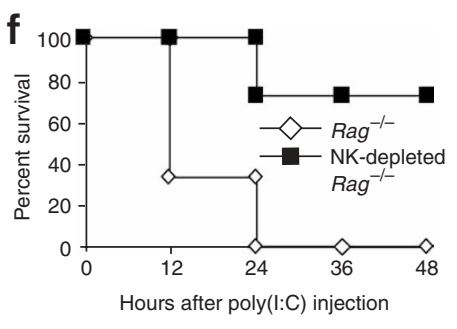

Figure 4 TNFR-hlg can substantially improve survival rates among nude and Rag-1 knockout mice treated with poly(I:C), and NK cells are critical in the sudden death of Rag-1 knockout mice after poly $(\mathrm{I}: \mathrm{C})$ injection. (a) T cells inhibited induction of TNF-producing $\mathrm{CD} 11 \mathrm{~b}^{+}$or $\mathrm{CD} 11 \mathrm{c}^{+}$cells and IFN- $\gamma-$ producing NK cells in vitro. (b) Decreased inflammatory cytokines from innate cells (CD11 b cells, NK cells or both) after poly (I:C) in the presence of T cells. (c) Mortality curve of control hlg-treated $(\diamond, n=10)$ and TNFR-hlg-treated nude mice $(\boldsymbol{\square}, n=5)$ after injection of $700 \mu \mathrm{g}$ poly(I:C). (d) Mortality curve of Rag-1 knockout mice (hlg: $\diamond, n=5$ and TNFR-hlg: $\mathbf{\square}, n=5$ ) after poly(I:C)
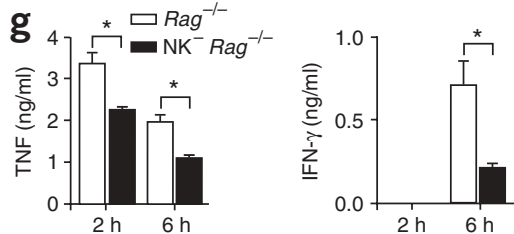

injection. (e) Decreased inflammatory cytokines from non-T cells after poly(I:C) stimulation and addition of soluble TNFR-hlg. (f) Mortality curve of Rag-1 knockout $(\diamond, n=8)$ and NK-depleted Rag-1 knockout $(\boldsymbol{\square}, n=8)$ mice. (g) Levels of proinflammatory cytokines in serum from Rag-1 knockout and NK-depleted $\left(\mathrm{NK}^{-}\right)$Rag-1 knockout mice were determined at 2 and $6 \mathrm{~h}$ after poly $(\mathrm{I}: \mathrm{C})$ injection. ${ }^{*} P<0.05,{ }^{* *} P<0.01$, by $t$-test.

\section{METHODS}

Mice. BALB/c mice and nude mice of BALB/c background were purchased from Vital River, and C57BL/6 Rag-1 knockout mice, C57BL/6J and MHC class II knockout mice were purchased from the Jackson Laboratory. FoxP3 (GFP) knock-in mice were a gift (see Acknowledgments). Animal care and experiwere performed in accordance with institutional and US National Institutes of Health guidelines and were approved by the animal use committee of the University of Chicago. Informed consent was obtained from all subjects for the use of human blood samples.

Virus, viral infection and viral titers. MHV-A59 virus and cell line were gifts (see Acknowledgments). Mice were infected intraperitoneally (i.p.) with $4 \times 10^{5}$ plaque-forming units (PFU) live virus. Viral titers were determined by plaque assay in $17 \mathrm{Cl}-1$ cells.

Poly(I:C) injection, TNF blockage by TNFR-human immunoglobulin (TNFR-hIg), and NK-cell depletion. Wild-type BALB/c mice and nude mice were injected i.p. with $700 \mu \mathrm{g}$ poly(I:C) and C57BL6 and Rag-1 knockout mice were injected i.p. with $400 \mu \mathrm{g}$ poly(I:C) (InvivoGen). Serum was collected at indicated time after injection. Two groups of nude or Rag-1 knockout mice were injected i.p. with $300 \mu \mathrm{g}$ control hIgG and TNFR-hIg, respectively, $2 \mathrm{~d}$ and $2 \mathrm{~h}$ before poly(I:C) injection. For depletion of NK cells, anti-NK1.1 or antiasialo-GM-1 was injected in Rag-1 knockout mice i.p. at day 4 and day 1 before poly(I:C) injection.

Isolation of splenocytes, non- $\mathrm{T}$, pan- $\mathrm{T}, \mathrm{CD}^{+}{ }^{+} \mathrm{T}$ cells, $\mathrm{CD8}^{+} \mathrm{T}$ cells, $\mathrm{CD25}{ }^{-}-$ depleted $\mathrm{T}$ cells and FoxP3 ${ }^{-} \mathrm{T}$ cell, and adoptive transfer of lymphocytes. Non- $\mathrm{T}$, pan- $\mathrm{T}, \mathrm{CD} 4^{+} \mathrm{T}$ and $\mathrm{CD} 8^{+} \mathrm{T}$ cells were isolated by MACS (Miltenyi Biotec). To deplete $\mathrm{CD} 25^{+} \mathrm{T}$ cells, the isolated pan-T cells were negatively selected after ligation with anti-CD25-phycoerythrin and anti-phycoerythrin microbeads. Pan-T cells were isolated from splenocytes of FoxP3(GFP) knock-in mice and then GFP-negative cells (non-Treg) and GFP-positive cells (Treg) were harvested by using MoFlow. Splenocytes and lymph node cells were harvested from wild-type C57BL6 mice and were incubated for $2 \mathrm{~h}$ at $37{ }^{\circ} \mathrm{C}$. The suspended cells were harvested and $300 \times 10^{6}$ cells transferred into knockout mice.

FACS analysis. Splenocytes or purified cells were stained with B220-fluorescein isothiocyanate (B220-FITC), DX5-biotin, CD11b-biotin, CD4-FITC and CD8-FITC (BD Pharmingen). To stain intracellular IFN- $\gamma$ and TNF, non-T cells were stimulated with poly(I:C) in the presence or absence of $\mathrm{T}$ cells and Golgi stop (BD Pharmingen) was added after $4 \mathrm{~h}$ of culture at $37^{\circ} \mathrm{C}$. The cells were incubated for $16 \mathrm{~h}$ under these condition and then stained with NK1.1allophycocyanin or CD11b and fixed. The fixed, permeabilized cells were stained with phycoerythrin-conjugated antibodies to mouse IFN- $\gamma$ or TNF. FACS data were analyzed with FlowJo software (Becton Dickinson).

Poly(I:C), MHV-A59 and LPS stimulation in vitro. Splenocytes from adult or neonatal mice, or T cell-depleted splenocytes, were stimulated with 50 or $100 \mu \mathrm{g} / \mathrm{ml}$ poly(I:C), $5 \times 10^{5} \mathrm{PFU}$ MHV-A59, or 100 or $500 \mathrm{ng} / \mathrm{ml}$ LPS in the presence or absence of different subsets of T cells. After coculture in a 96-well round-bottom plate in MEM medium for $20 \mathrm{~h}$, supernatants were collected for cytokine analysis. A Transwell plate system (Costar; pore size $0.4 \mu \mathrm{m})$ was used to prevent close contact between non-T cells and $\mathrm{T}$ cells.

Analysis of cytokine production. The amount of cytokines was quantified using the cytometric bead array kit for mouse inflammatory cytokines (CBA; BD Biosciences) on a FACSCalibur cytometer equipped with CellQuestPro and CBA software (Becton Dickinson).

Statistical analysis. The Mann-Whitney test (for survival) and Student's $t$-test (for cytokines) were used. Error bars represent s.d. or s.e.m. 


\section{LETTERS}

Note: Supplementary information is available on the Nature Medicine website.

\section{ACKNOWLEDGMENTS}

We would like to acknowledge L. Su, H. Deng, X. Shi and Y. Liu for productive discussion and suggestions. We thank C.-R. Wang (University of Chicago) for MHC class II-deficient mice (originally from Jackson Laboratory), A.Y. Rudensky (Univ. Washington) for FoxP3 (GFP) knock-in mice and R. Baric (Univ. North Carolina) for the MHV-A59 virus and cell line. This research was in part supported by US National Institutes of Health grants AI062026, CA115540 and DK58891 to Y.X.F. and by a National Science Foundation of China grant (30430640) and Ministry of Science and Technology grants (2002CB513000, (2) 2004BA519A61, 2006CB504300) to H.T. S.A. is part of the Medical Scientist $\frac{.}{0}$ Training Program at the University of Chicago and is supported by a Medical Scientist National Research Service Award (5 T32 GM07281)

\section{AUTHOR CONTRIBUTIONS}

K.D.K. and J.Z. conducted most of the experiments. S.A., X.Y. and P.D. provided technical support. S.A. and H.T. edited the paper. H.T. and Y.-X.F. organized and supervised the project. K.D.K. and Y.-X.F. wrote the manuscript.

Published online at http://www.nature.com/naturemedicine

Reprints and permissions information is available online at http://npg.nature.com/ reprintsandpermissions

1. Medzhitov, R. \& Janeway, C.A. Jr. Innate immunity: the virtues of a nonclonal system of recognition. Cell 91, 295-298 (1997).

2. Poltorak, A. et al. Defective LPS signaling in $\mathrm{C} 3 \mathrm{H} / \mathrm{HeJ}$ and $\mathrm{C} 57 \mathrm{BL} / 10 \mathrm{ScCr}$ mice: mutations in TIr4 gene. Science 282, 2085-2088 (1998).

3. Gazzinelli, R.T. \& Denkers, E.Y. Protozoan encounters with Toll-like receptor signalling pathways: implications for host parasitism. Nat. Rev. Immunol. 6, 895-906 (2006).

4. Trinchieri, G. \& Sher, A. Cooperation of Toll-like receptor signals in innate immune defence. Nat. Rev. Immunol. 7, 179-190 (2007).
5. Lavi, E., Gilden, D.H., Highkin, M.K. \& Weiss, S.R. Persistence of mouse hepatitis virus A59 RNA in a slow virus demyelinating infection in mice as detected by in situ hybridization. J. Virol. 51, 563-566 (1984).

6. Haring, J. \& Perlman, S. Mouse hepatitis virus. Curr. Opin. Microbiol. 4, 462-466 (2001).

7. Schroder, M. \& Bowie, A.G. TLR3 in antiviral immunity: key player or bystander? Trends Immunol. 26, 462-468 (2005).

8. Proft, T. \& Fraser, J.D. Bacterial superantigens. Clin. Exp. Immunol. 133, 299-306 (2003).

9. Pfeffer, K. et al. Mice deficient for the $55 \mathrm{kd}$ tumor necrosis factor receptor are resistant to endotoxic shock, yet succumb to L. monocytogenes infection. Cell $\mathbf{7 3}$, 457-467 (1993).

10. Glauser, M.P., Zanetti, G., Baumgartner, J.D. \& Cohen, J. Septic shock: pathogenesis. Lancet 338, 732-736 (1991).

11. Maloy, K.J. et al. $\mathrm{CD} 4^{+} \mathrm{CD} 25^{+} \mathrm{T}(\mathrm{R})$ cells suppress innate immune pathology through cytokine-dependent mechanisms. J. Exp. Med. 197, 111-119 (2003).

12. Kim, J.M., Rasmussen, J.P. \& Rudensky, A.Y. Regulatory T cells prevent catastrophic autoimmunity throughout the lifespan of mice. Nat. Immunol. 8, 191-197 (2007).

13. Fontenot, J.D. et al. Regulatory T cell lineage specification by the forkhead transcription factor foxp3. Immunity 22, 329-341 (2005).

14. Andoniou, C.E. et al. Interaction between conventional dendritic cells and natural killer cells is integral to the activation of effective antiviral immunity. Nat. Immunol. 6 1011-1019 (2005)

15. Andrews, D.M., Scalzo, A.A., Yokoyama, W.M., Smyth, M.J. \& Degli-Esposti, M.A Functional interactions between dendritic cells and NK cells during viral infection. Nat. Immunol. 4, 175-181 (2003).

16. Fernandez, N.C. et al. Dendritic cells directly trigger NK cell functions: cross-talk relevant in innate anti-tumor immune responses in vivo. Nat. Med. 5, 405-411 (1999).

17. Munz, C., Steinman, R.M. \& Fujii, S. Dendritic cell maturation by innate lymphocytes: coordinated stimulation of innate and adaptive immunity. J. Exp. Med. 202, 203-207 (2005).

18. Vitale, M. et al. NK-dependent DC maturation is mediated by TNF $\alpha$ and IFN $\gamma$ released upon engagement of the NKp30 triggering receptor. Blood 106, 566-571 (2005).

19. El-Sawy, T., Miura, M. \& Fairchild, R. Early T cell response to allografts occurring prior to alloantigen priming up-regulates innate-mediated inflammation and graft necrosis. Am. J. Pathol. 165, 147-157 (2004). 